УДК 532.516

\author{
А. В. Зинченко*, С. С. Мирный* \\ *Институт транспортных систем и технологий НАН Украины
}

\title{
О ВЗАИМНОМ ВЛИЯНИИ РАСПОЛОЖЕНЫХ В РЯД ЦИЛИНДРОВ В ПОТОКЕ ВЯЗКОЙ НЕСЖИМАЕМОЙ ЖИДКОСТИ
}

Работа посвящена исследованию внешнего обтекания групп бесконечных цилиндров вязкой несжимаемой жидкостью, определению влияния расстояния между центрами цилиндров в группе на их аэродинамические характеристики и общую картину течения. Выполнено численное моделирование обтекания группы из трех цилиндров на основе решения уравнений Навье-Стокса. Параметры потока были выбраны соответствующими числу Рейнольдса 120 при обтекании одиночного цилиндра. Расстояния между центрами соседних цилиндров варьировались в диапазоне от 1.1 до 6.0 диаметров цилиндра. Выделены три характерные картины течения в зависимости от расстояния между центрами цилиндров. Первая - полностью стационарное течение. Данная картина течения наблюдается при расстоянии между центрами цилиндров менее трех диаметров. При этом группа цилиндров обтекается как единое целое. Вторая характерная картина наблюдается при расстоянии между центрами, равном четырем диаметрам. Для этого случая течение между цилиндрами стационарное, а за группой формируется вихревая дорожка. Третья характерная картина течения наблюдается при дальнейшем увеличении расстояния между цилиндрами. Течение между цилиндрами также становится нестационарным. Получены осредненные коэффициенты лобового сопротивления для каждого из цилиндров в группе. Во всех рассматриваемых конфигурациях коэффициенты лобового сопротивления всех цилиндров по абсолютной величине меньше осредненного коэффициента лобового сопротивления одиночного цилиндра. Для первой и второй картины течения коэффициент лобового сопротивления центрального цилиндра отрицателен.

Ключевые слова: численное моделирование, вычислительная гидродинамика, обтекание группы тел, уравнения Навье-Стокса.

Робота присвячена дослідженню зовнішнього обтікання груп нескінчених циліндрів в'язкою нестисливою рідиною, визначенню впливу відстані між центрами циліндрів у групі на їх аеродинамічні характеристики та загальну картину течії. Було проведено чисельне моделювання обтікання групи 3 трьох циліндрів на основі рівнянь Нав'є -Стокса. Параметри потоку були вибрані у відповідності до числа Рейнольдса 120 при обтіканні одиничного циліндра. Відстані між центрами сусідніх циліндрів варіювалися у діапазоні від 1.1 до 6.0 діаметрів циліндра. У залежності від відстані було виявлено три характерні картини течії. Перша - повністю стаціонарна течія, така картина спостерігається при відстані між центрами циліндрів менше трьох діаметрів циліндра. При цьому група циліндрів обтікається рідиною як єдине ціле. Друга характерна картина спостерігається при відстані між центрами циліндрів, що дорівнює чотирьом діаметрам циліндра. У цьому випадку течія між циліндрами с стаціонарною, а за групою формусться вихорова доріжка. Третя картина течії спостерігається при подальшому збільшенні відстані. Течія між циліндрами теж набуває нестаціонарного характеру. Отримано усереднені коефіціснти лобового опору для кожного 3 циліндрів у групі. В усіх досліджених конфігураціях коефіціснти лобового опору усіх циліндрів за абсолютною величиною менше осередненого коефіцієнту лобового опору одиничного циліндра. Для першої та другої картини течії коефіцієнт лобового опору центрального циліндра с від'ємним.

Ключові слова: чисельне моделювання, обчислювальна гідродинаміка, обтікання групи тіл, рівняння Нав'є-Стокса.

The paper is devoted to the study of viscous incompressible flow around a group of infinite cylinders. The influence of the distance between the centers of the cylinders in the group on their aerodynamic characteristics and the general flow pattern is investigated. A numerical simulation of the flow around a group of three cylinders based on solving the Navier-Stokes equations is performed. The

(C) Зинченко А. В., Мирный С. С., 2019 
flow parameters were chosen corresponding to the Reynolds number 120 for standalone cylinder. The distances between the centers of neighboring cylinders ranged from 1.1 to 6.0 cylinder's diameters. Three characteristic flow patterns are distinguished depending on the distance between the cylinders. The first is a fully stationary flow. This flow pattern is formed when the distance between the cylinders is below three diameters. The flow pattern looks similar to the flow around the standalone cylinder. The second flow pattern is observed at a distance between the centers of four diameters. For this case, the flow between the cylinders remains stationary, but the vortex street is formed behind the group. The third flow pattern is observed with further increasing the distance between the cylinders. The flow between the cylinders becomes unsteady as well. The averaged drag coefficients for each of the cylinders in the group are calculated. In all configurations considered the absolute value of the drag coefficients of all cylinders are less than the average drag coefficient of standalone cylinder. For the first and second flow patterns, the drag coefficient of the central cylinder is negative.

Keywords: numerical simulation, CFD, flow around a group of bodies, Navier-Stokes equations.

Введение. Исследование обтекания групп тел потоком газа или жидкости является важной задачей современной механики жидкости и газа. Особенно важны исследования влияния взаимного положения тел в потоке при нестационарном обтекании группы тел. В этом случае нестационарные и осредненные по времени аэродинамические характеристики могут изменяться в значительной степени $[2,6,7$, $8,11]$.

Настоящее исследование посвящено определению влияния расстояния между центрами цилиндров на их аэродинамические характеристики и картину течения. Основным методом исследования в данной работе является математическое моделирование с помощью численного решения уравнений Навье-Стокса. Для решения уравнений Навье-Стокса разработано большое количество численных методов $[3,4,5,9]$. В данной работе для численного решения уравнений, описывающих течение жидкости, используется метод конечных объемов.

Обтекание одиночного цилиндра является достаточно изученной задачей. Моделирование обтекания группы цилиндров проводилось при таких параметрах набегающего потока, которые соответствуют параметрам потока, при котором число Рейнольдса при обтекании одиночного цилиндра равно 120, а число Маха не превышало 0.2. Данное число Рейнольдса соответствует ламинарному нестационарному обтеканию одиночно цилиндра. Так как число Маха не превышало 0.2 , то эффектами сжимаемости можно было пренебречь и среду в расчетной области можно было считать несжимаемой.

1. Физическая постановка задачи. В современной технике достаточно часто необходимо проанализировать взаимное влияние тел, расположенных близко в потоке. Такие конфигурации расположения тел в группе, в которых тела в потоке находятся одно за другим, достаточно часто встречаются в автомобильном и железнодорожном транспорте. На рис. 1 показана схема расположения цилиндров в группе.
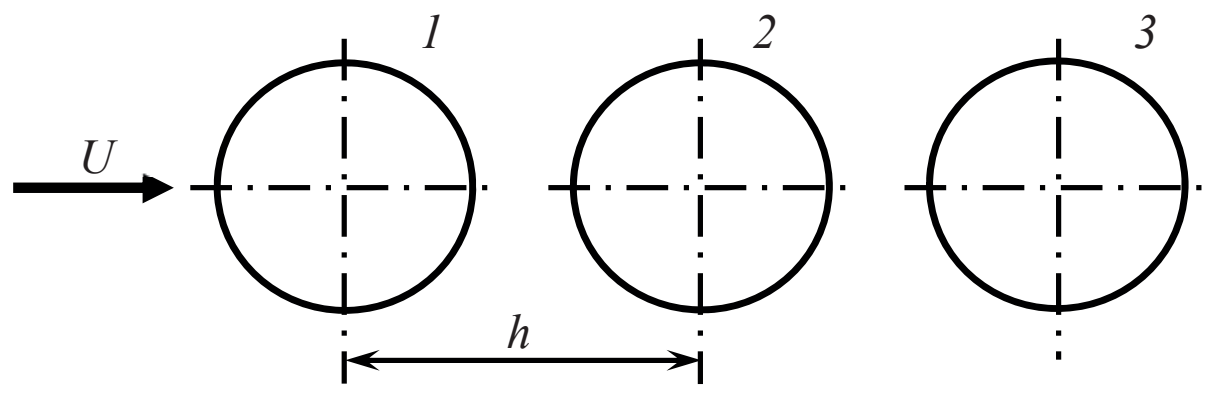

Рис. 1. Схема расположения цилиндров в группе 
При внешнем обтекании одиночного длинного цилиндра при отсутствии теплообмена основным безразмерным параметром является число Рейнольдса Re. Известно, что при обтекании одиночного цилиндра можно выделить несколько режимов течения $[1,6]$. При числе Рейнольдса меньше 5 течение является стационарным и безотрывным, При $5<R e<40$ течение стационарное, за цилиндром возникает два симметричных вихря. При числе Рейнольдса от 40 до 200 на цилиндре формируется ламинарный пограничный слой, происходит периодический отрыв вихрей и формируется ламинарная вихревая дорожка Кармана. При дальнейшем увеличении числа Рейнольдса до 300 происходит переход к турбулентному течению в следе за цилиндром. При числе Рейнольдса от 300 до $3 \cdot 10^{5}$ на цилиндре формируется ламинарный пограничный слой, но при этом течение в следе за цилиндром полностью турбулентное и такой режим течения называется докритическим. При $3 \cdot 10^{5}<\operatorname{Re}<4.5 \cdot 10^{5}$ происходит резкое падение коэффициента лобового сопротивления и данное течение является критическим. При числе Рейнольдса больше $4.5 \cdot 10^{5}$ течение становится закритическим. Происходит постепенное увеличение коэффициента лобового сопротивления.

В данном исследовании при моделировании обтекания группы из трех цилиндров параметры потока выбирались соответствующими числу Рейнольдса 120 при обтекании одиночного цилиндра такого же диаметра. Расстояния между центрами соседних цилиндров $\mathrm{h}$ равнялись $1.1,1.5,2.0,3.0,4.0,5.0,6.0$ диаметров цилиндра.

2. Математическая модель обтекания группы цилиндров. Математическое моделирование проводилось с помощью численного решения уравнений НавьеСтокса для ламинарного течения несжимаемой жидкости в консервативной форме $[3,4,5]:$

$$
\begin{gathered}
\nabla \cdot \vec{V}=0 \\
\frac{\partial \vec{V}}{\partial t}+\nabla \cdot(\vec{V} \cdot \vec{V})+\frac{\nabla p}{\rho}=v \cdot \nabla^{2} \vec{V}
\end{gathered}
$$

где $\vec{V}$ - вектор скорости, $p$ - давление, $v$ - динамическая вязкость, $\rho$ - плотность жидкости. Уравнение (1) представляет собой уравнение неразрывности, а уравнение (2) является уравнением сохранения количества движения.

Для двумерного случая уравнения Навье-Стокса несжимаемой жидкости в консервативной форме можно записать в следующем виде $[4,5]$ :

$$
\begin{gathered}
\frac{\partial u}{\partial x}+\frac{\partial v}{\partial y}=0 \\
\frac{\partial u}{\partial t}+\frac{\partial(u \cdot u)}{\partial x}+\frac{\partial(v \cdot u)}{\partial y}=-\frac{1}{\rho} \cdot \frac{\partial p}{\partial x}+v \cdot\left(\frac{\partial^{2} u}{\partial x^{2}}+\frac{\partial^{2} u}{\partial y^{2}}\right) \\
\frac{\partial v}{\partial t}+\frac{\partial(u \cdot v)}{\partial x}+\frac{\partial(v \cdot v)}{\partial y}=-\frac{1}{\rho} \cdot \frac{\partial p}{\partial y}+v \cdot\left(\frac{\partial^{2} v}{\partial x^{2}}+\frac{\partial^{2} v}{\partial y^{2}}\right)
\end{gathered}
$$

где $u$ - проекция вектора скорости жидкости $\vec{V}$ на ось $O x, v$ - проекция вектора скорости $\vec{V}$ на ось $O y$. 
Особенностью уравнений Навье-Стокса для несжимаемой жидкости является отсутствие производной давления по времени в уравнении неразрывности, что приводит к тому, что поля давления и скоростей не связаны между собой. Для согласования между собой полей скоростей и давления будем использовать метод искусственной сжимаемости [5].

В методе искусственной сжимаемости в уравнение неразрывности (3) добавляется частная производная давления по времени и вводится коэффициент искусственной сжимаемости. Таким образом, уравнения (3) - (5) можно записать следующим образом:

$$
\begin{aligned}
& \frac{\partial p}{\partial t}+\beta \cdot\left(\frac{\partial u}{\partial x}+\frac{\partial v}{\partial y}\right)=0, \\
& \frac{\partial u}{\partial t}+\nabla(\vec{V} \cdot u)=-\frac{1}{\rho} \frac{\partial p}{\partial x}+v \cdot \nabla(\nabla u), \\
& \frac{\partial v}{\partial t}+\nabla(\vec{V} \cdot v)=-\frac{1}{\rho} \frac{\partial p}{\partial y}+v \cdot \nabla(\nabla v) .
\end{aligned}
$$

Для получения дискретных аналогов пространственных производных в уравнениях использовался метод конечных объемов $[3,4,5]$. Аппроксимации конвективных слагаемых на грани конечных объемов выполнялась с использованием противопоточной схемы второго порядка. Для аппроксимации вязких слагаемых использовалась теорема Гаусса-Остроградского. Для получения дискретных аналогов производных по времени использовался метод КранкаНиколсона [5]. Метод Кранка-Николсона является неявным, второго порядка точности и обеспечивает большую устойчивость решения, что позволяет значительно увеличить шаг по времени при проведении расчетов. Таким образом, обеспечивается сокращение общего времени вычислений по сравнению со временем, которое необходимо для математического моделирования с использованием явных численных методов.

3. Моделирование обтекания группы из трех цилиндров. Моделирование обтекания группы цилиндров проводилось с помощью численного решения системы уравнений (6) - (8) в среде пакета программ с открытым исходным кодом OpenFOAM v.1810 [10].

В табл. 1 и на рис. 2 приведены осредненные коэффициенты лобового сопротивления для трех цилиндров в группе.

Таблица 1

Осредненные коэффициенты лобового сопротивления цилиндров в группе

\begin{tabular}{|l|l|l|l|l|}
\hline $\mathrm{h}$ & $\mathrm{Cx} 1$ & $\mathrm{Cx} 2$ & $\mathrm{Cx} 3$ & Режим \\
\hline 1.1 & 1.184 & -0.145 & 0.0102 & стационарный \\
\hline 1.5 & 1.164 & -0.153 & 0.0565 & стационарный \\
\hline 2.0 & 1.146 & -0.157 & 0.1075 & стационарный \\
\hline 3.0 & 1.113 & -0.1404 & 0.1943 & стационарный \\
\hline 4.0 & 1.093 & -0.1 & 0.2879 & нестационарный \\
\hline 5.0 & 1.1422 & 0.35 & 0.4196 & нестационарный \\
\hline 6.0 & 1.1556 & 0.4586 & 0.312 & нестационарный \\
\hline
\end{tabular}




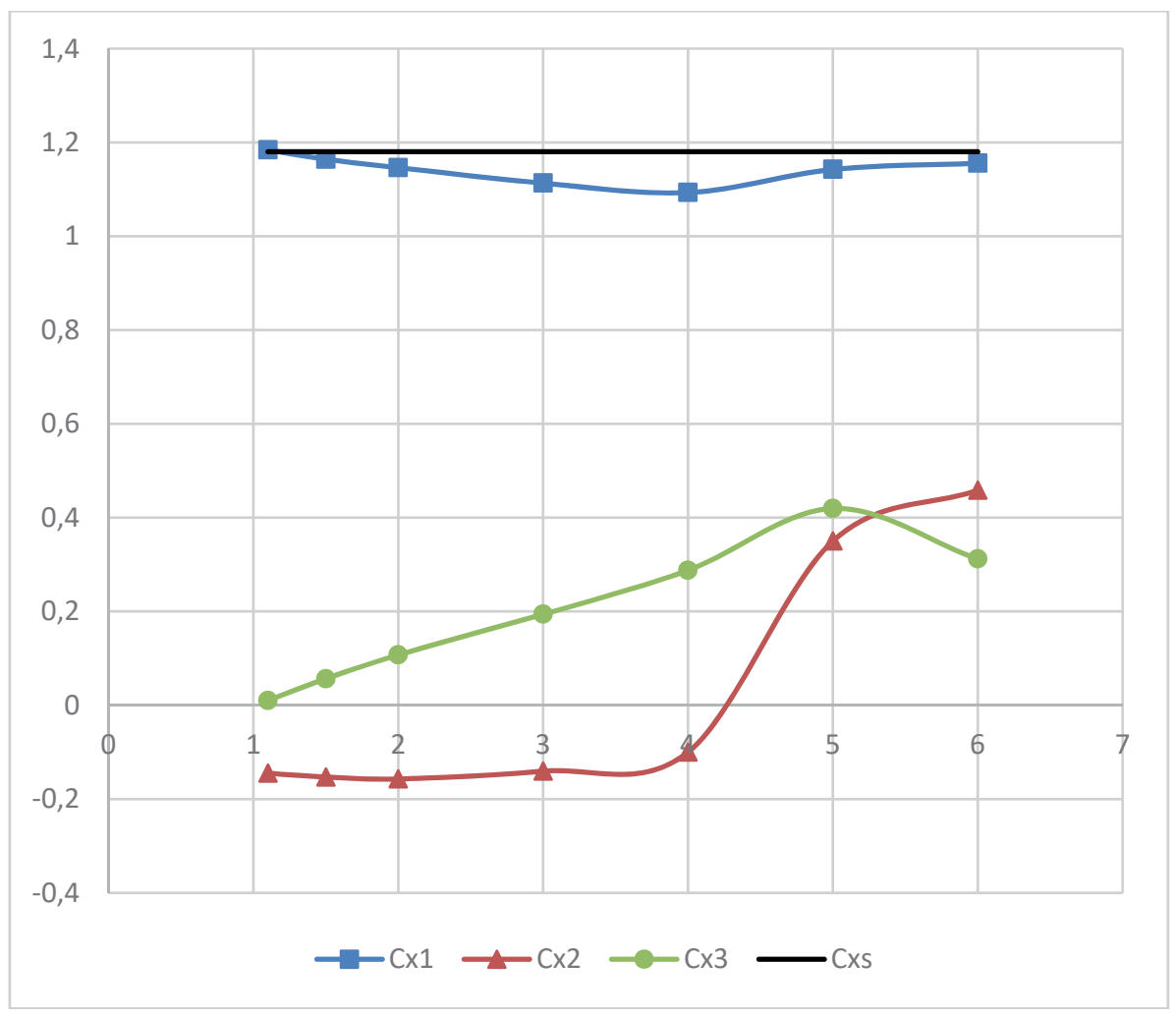

Рис. 2. Осредненные коэффициенты лобового сопротивления: Сх1 - первого цилиндра, Cx2 - второго цилиндра, Cx3 - третьего цилиндра, Cxs - одиночного цилиндра

На рис. 3 - 5 приведены мгновенные распределения безразмерного вектора скорости.

Для расстояний между центрами цилиндров до $3.0 \mathrm{D}$ течение стационарное и группа цилиндров обтекается как единое удлиненное тело (рис.3). При этом коэффициент лобового сопротивления второго цилиндра отрицателен. Это объясняется наличием стационарных вихрей между первым и вторым и между вторым и третьим цилиндрами, а также тем, что давление за вторым цилиндром выше, чем перед вторым цилиндром.

Для расстояния между цилиндрами $4.0 \mathrm{D}$ течение между цилиндрами все еще стационарное, но за группой формируется вихревая дорожка (рис. 4). Коэффициент лобового сопротивления второго цилиндра отрицателен, так как давление за ним все еще выше, чем перед ним.

При дальнейшем увеличении расстояния между центрами цилиндров происходит перестройка течения между цилиндрами, и течение становится полностью нестационарным (рис. 5). Коэффициенты лобового сопротивления цилиндров становятся положительными.

Во всех рассматриваемых случаях коэффициенты лобового сопротивления для всех цилиндров по абсолютной величине меньше осредненного коэффициента лобового сопротивления одиночного цилиндра. 


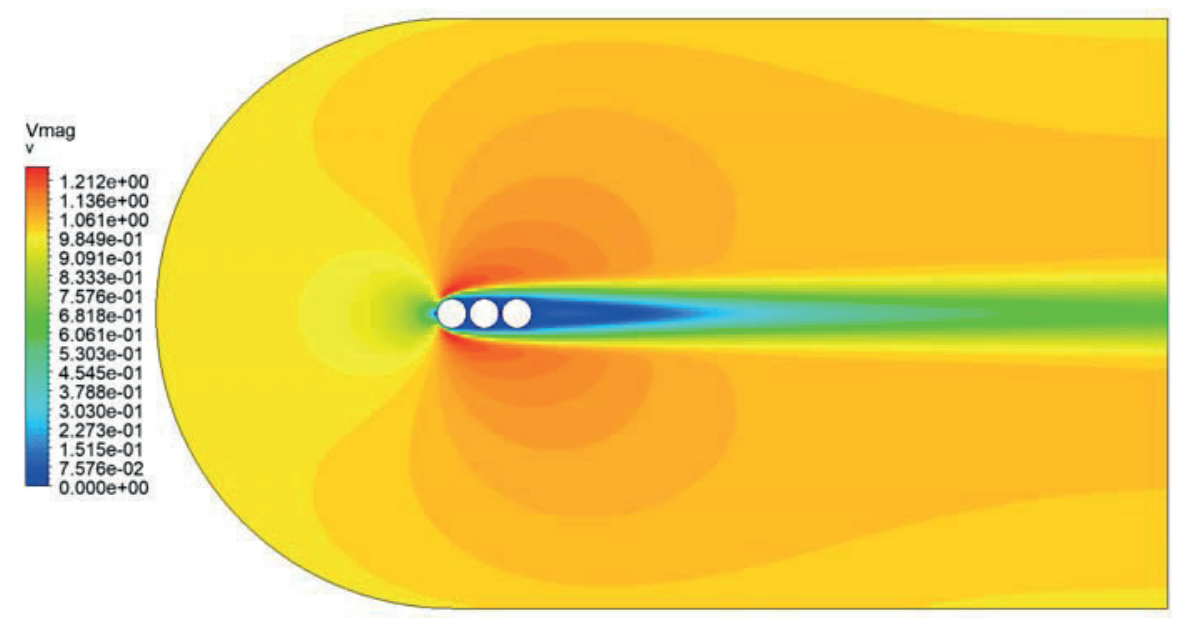

Рис. 3. Мгновенное распределение длины вектора скорости при $\mathrm{h}=1.1$

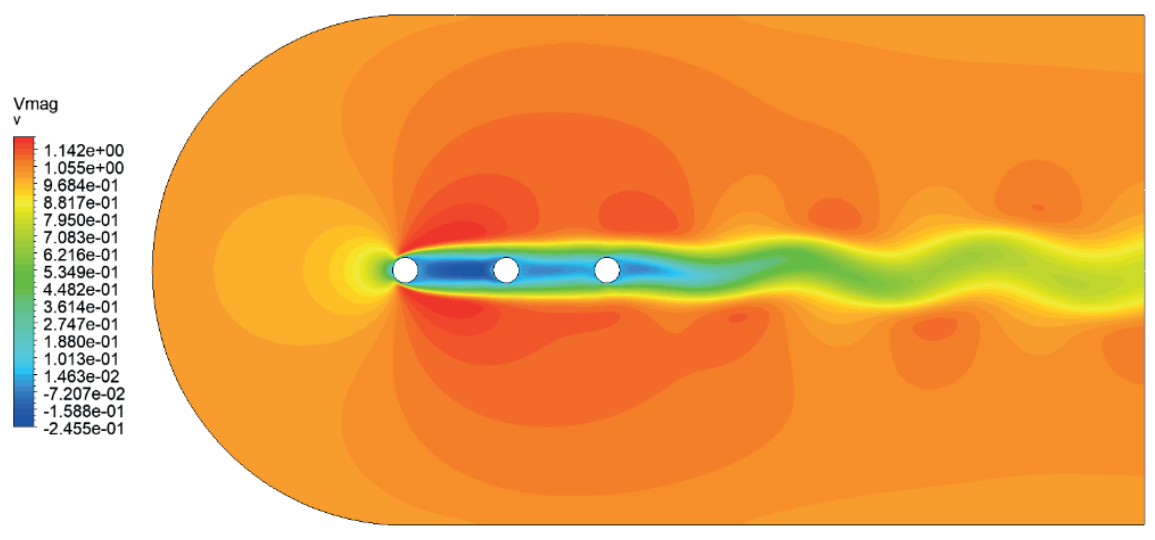

Рис. 4. Мгновенное распределение длины вектора скорости при $\mathrm{h}=4.0$

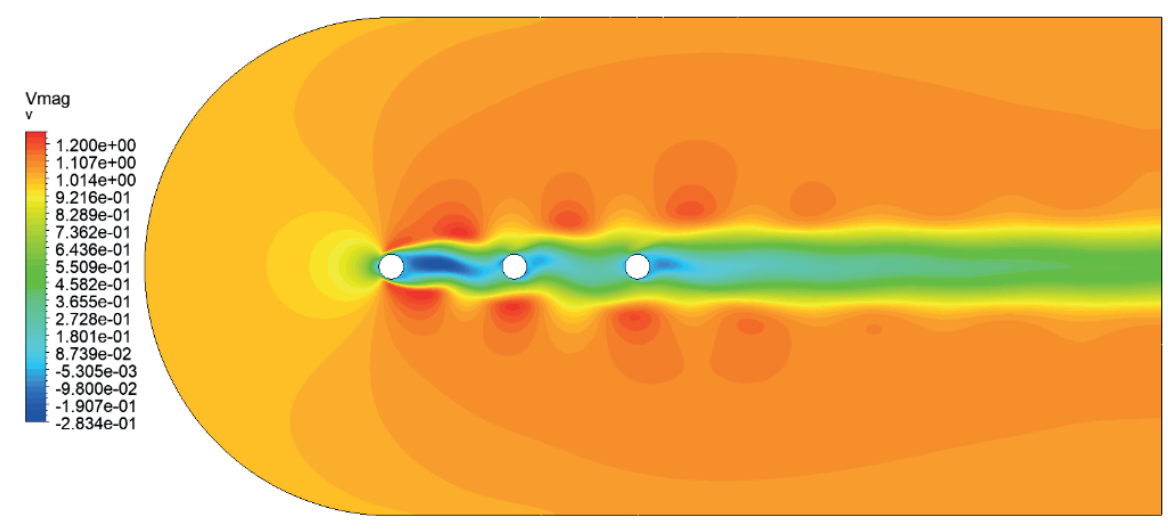

Рис. 5. Мгновенное распределение длины вектора скорости при $\mathrm{h}=\mathbf{5 . 0}$ 
Заключение. Течение при обтекании группы из трех цилиндров имеет сложную структуру, и соответствующая картина течения зависит от расстояния между центрами цилиндров.

Можно выделить три характерные картины течения. Первая - это полностью стационарное течение. Данная картина течения наблюдается при расстоянии между центрами цилиндров меньше $3.0 \mathrm{D}$. При этом группа цилиндров обтекается как единое целое. Вторая характерная картина наблюдается при расстоянии между центрами равном $4.0 \mathrm{D}$. Для этого случая течение между цилиндрами стационарное, а за группой формируется вихревая дорожка. Третья характерная картина течения наблюдается при дальнейшем увеличении расстояния между цилиндрами. Течение между цилиндрами также становится нестационарным.

Для всех случаев коэффициенты лобового сопротивления цилиндров меньше, чем коэффициент лобового сопротивления одиночного цилиндра. Для первой и второй картины течения коэффициент лобового сопротивления центрального цилиндра отрицателен. Это объясняется тем, что давление за центральным цилиндром больше, чем перед ним.

\section{Библиографические ссылки}

1. Лойцянский, Л. Г. Механика жидкости и газа / Л. Г. Лойцянский. - М: Дрофа - 2003.

2. Carmo, B. S. On Wake Interference in the Flow around Two Circular Cylinders: Direct Stability Analysis and Flow-Induced Vibrations / B. S. Carmo // PhD thesis. - Department of Aeronautics, Imperial College, London. - 2009. - 262 p.

3. Chung, T. J. Computational Fluid Dynamics, 2nd ed. / T. J. Chung. - New York City, USA: Cambridge University Press, 2009. - 1058 p.

4. Ferziger, J. H. Computational Methods for Fluid Dynamics / J. H. Ferziger, M. Peric. Springer. $-2002 .-431 \mathrm{p}$.

5. Hoffmann, K. A. Computational Fluid Dynamics, 4th ed. V1 / K. A. Hoffmann, S.T. Chiang. - Wichita, Kansas, USA: Engineering Education System, 2000. - 500 p.

6. Kaneko, S. Flow-Induced Vibrations: Classifications and Lessons from Practical Experiences / S. Kaneko. - Elsevier. - 2014. - 411 p.

7. Kim, H. J. Investigation of the flow between a pair of circular cylinders in the flopping regime / H. J. Kim, P. A. Durbin // J. Fluid Mech. - 1988. - Vol. 196. - P. 431-448.

8. Sumner, D. Two staggered circular cylinders of equal diameter in cross-flow / D. Sumner, M. D. Richards, O. O. Akosile. // Journal of Fluids and Structures. - 2005. - № 20. - P. 255-276.

9. Versteeg, H. K. An Introduction to Computational Fluid Dynamics / H. K. Versteeg, W. Malalasekera. - England, Harlow: Pearson Education Limited. - 2007. - 517 p.

10.Weller, H. G. A tensorial approach to computational continuum mechanics using objectoriented techniques / H. G. Weller, G. Tabor, H. Jasak, C. Fureby // Computers in Physics. Nov/Dec 1998. - Vol. 12, № 6.

11.Williamson, C. H. K. Evolution of a single wake behind a pair of bluff bodies / C. H. K. Williamson // J. Fluid Mech. - 1985. - Vol. 159. - P. 1-18. 